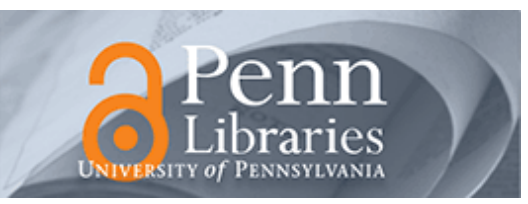

University of Pennsylvania

ScholarlyCommons

6-8-2003

\title{
Radiation Characteristics of Microstrip Dipole Antennas over a High-Impedance Metamaterial Surface made of Hilbert Inclusions
}

\author{
John McVay \\ Villanova University \\ Ahmad Hoorfar \\ Villanova University \\ Nader Engheta \\ University of Pennsylvania, engheta@seas.upenn.edu
}

Follow this and additional works at: https://repository.upenn.edu/ese_papers

Part of the Electrical and Computer Engineering Commons

\section{Recommended Citation \\ John McVay, Ahmad Hoorfar, and Nader Engheta, "Radiation Characteristics of Microstrip Dipole Antennas over a High-Impedance Metamaterial Surface made of Hilbert Inclusions", . June 2003.}

Copyright 2003 IEEE. Reprinted from IEEE MTT-S International Microwave Symposium Digest 2003 Volume 1, pages 587-590.

Publisher URL: http://ieeexplore.ieee.org/xpl/tocresult.jsp?isNumber=27238\&page=9

This material is posted here with permission of the IEEE. Such permission of the IEEE does not in any way imply IEEE endorsement of any of the University of Pennsylvania's products or services. Internal or personal use of this material is permitted. However, permission to reprint/republish this material for advertising or promotional purposes or for creating new collective works for resale or redistribution must be obtained from the IEEE by writing to pubs-permissions@ieee.org. By choosing to view this document, you agree to all provisions of the copyright laws protecting it.

This paper is posted at ScholarlyCommons. https://repository.upenn.edu/ese_papers/10

For more information, please contact repository@pobox.upenn.edu. 


\title{
Radiation Characteristics of Microstrip Dipole Antennas over a High-Impedance Metamaterial Surface made of Hilbert Inclusions
}

\author{
Abstract \\ We analyze numerically the radiation characteristics of center-fed microstrip short dipoles and half-wave \\ dipoles near a high-impedance surface made of Hilbert shape flat inclusions. We study the input \\ impedance, pattern and gain of such radiating structures. We show that the radiation resistance of a \\ microstrip dipole increases noticeably at certain frequencies near the resonant frequency of the Hilbert \\ surface. Moreover, antenna gain enhancement at certain frequencies is observed for all dipole sizes we \\ have analyzed.

\section{Disciplines} \\ Electrical and Computer Engineering

\section{Comments} \\ Copyright 2003 IEEE. Reprinted from IEEE MTT-S International Microwave Symposium Digest 2003 \\ Volume 1, pages 587-590. \\ Publisher URL: http://ieeexplore.ieee.org/xpl/tocresult.jsp?isNumber=27238\&page=9 \\ This material is posted here with permission of the IEEE. Such permission of the IEEE does not in any way \\ imply IEEE endorsement of any of the University of Pennsylvania's products or services. Internal or \\ personal use of this material is permitted. However, permission to reprint/republish this material for \\ advertising or promotional purposes or for creating new collective works for resale or redistribution must \\ be obtained from the IEEE by writing to pubs-permissions@ieee.org. By choosing to view this document, \\ you agree to all provisions of the copyright laws protecting it.
}




\title{
Radiation Characteristics of Microstrip Dipole Antennas over a High- Impedance Metamaterial Surface made of Hilbert Inclusions
}

\author{
John McVay ${ }^{1}$, Ahmad Hoorfar' ${ }^{1}$, and Nader Engheta ${ }^{2}$ \\ 'Villanova University, Department of Electrical and Computer Engineering, Villanova, PA, 19085 \\ ${ }^{2}$ University of Pennsylvania, Department of Electrical and Systems Engineering, Philadelphia, PA \\ 19104 \\ E-mail: john.mcvay@villanova.edu, hoorfar@ece.villanova.edu, engheta@ee.upenn.edu
}

\begin{abstract}
We analyze numerically the radiation characteristics of center-fed microstrip short dipoles and half-wave dipoles near a high-impedance surface made of Hilbert shape flat inclusions. We study the input impedance, pattern and gain of such radiating structures. We show that the radiation resistance of a microstrip dipole increases noticeably at certain frequencies near the resonant frequency of the Hilbert surface. Moreover, antenna gain enhancement at certain frequencies is observed for all dipole sizes we have analyzed.
\end{abstract}

\section{INTRODUCTION}

Design of low-profile, embedded, flush-mounted antennas are of paramount importance in a variety of applications in military and commercial domains. Since the platforms over which such antennas are placed are usually made of conducting structures, the interaction of antennas with these platforms should be taken into account. High impedance surfaces are planar metamaterial media composed of inclusions that need not be electrically small. At certain frequencies, such a surface may effectively act as an artificial magnetic conductor, which has a reflection coefficient of $R=+1$ for a plane wave incident on it, in contrast to the conventional electric conductor for which $R=-1$. These structures can therefore offer interesting applications in antenna design. For example, as originally shown by Sievenpiper et al. [1] for a surface with $R=+1$, the horizontal wire antennas can be laid near the surface, and the image current will be in phase with the antenna current, resulting in good radiation performance. Consequently microstrip and other printed antennas may benefit if their conventional highly conducting '(lowimpedance) ground-planes are replaced by highimpedance surfaces.

Various high impedance ground planes (HIGP) have been studied by several groups (see e.g., [1]-[3]). Recently we have shown that planar surfaces composed of inclusion cells patterned according to the Hilbert curve- filling algorithm, also manifest high-surface-impedance behavior (see Figure 1) [4]. The Hilbert curve is a member of the family of curves known in the mathematics literature as space-filling curves [5]. One of the interesting features of the Hilbert curve is the fact that as one considers higher step-orders of this curve, a long "line" can be compacted into a small "surface" area. As a result, the Hilbert curve offers a resonant structure that could have a relatively. very small footprint at a curve-filling iteration order $n=3$ or higher. The small footprint of Hilbert-curve cells makes them particularly attractive when a high density of inclusions is required. With a proper choice of the shape, dimensions and the periodicity of the inclusions one can achieve high surface impedance at a given resonance frequency and scan angle.

In this work we have investigated the radiation performance of microstrip antennas above a Hilbert-curve metamaterial surface near a conducting ground plane. In particular, method of moments (MOM) has been used to numerically model a short as well as a center-fed halfwave microstrip dipole above this structure. An extensive parametric study of the antenna input impedance, pattern and gain as a function of frequency is performed. In particular, it is found that the radiation resistance of a short dipole can be significantly increased resulting in an improved efficiency. In addition, a significant increase in antenna gain is achieved at specific frequencies for all dipole sizes analyzed.

In the following, we first present some results of the plane-wave scattering from a Hilbert-curve metamaterial surface over a ground plane. We then discuss some of the numerical results obtained for radiation of microstrip dipoles above such a structure.

\section{NUMERICAL RESULTS}

\section{A. Scattering from Hilbert-Curve Metamaterial Surface above a Conducting Ground Plane}

Consider the metamaterial surface, shown in Figure 1, for which many inclusions in the shape of the "Hilbert Curve" of step-order 3 are placed, in a 2-D periodic 
arrangement, close to a highly conducting ground plane. We have used a MOM based code with periodic boundary conditions to numerically analyze the scattering from this surface when illuminated with a plane-wave [4]. Figure 2 shows, the magnitude and phase of the reflection coefficient, $R$, for the case of a normal incidence wave polarized in $+x$ direction. As can be seen, at the frequency of $f=32.66 \mathrm{GHz}$, the phase of $\mathrm{R}$ is approximately zero. Since the amplitude of $R$ is one, at this frequency the surface acts as a high-impedance surface.

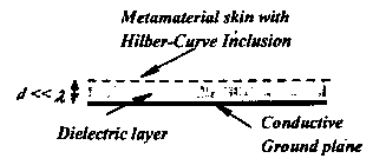

Side View
TopView

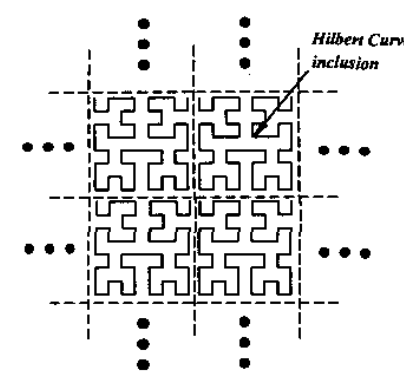

Fig. 1. Geometry of the Hilbert-curve metamaterial surface above a conducting ground plane. The Hilbert curve of step order 3 is shown here.

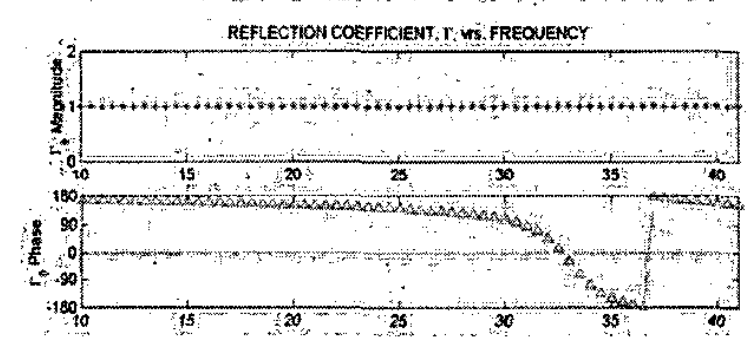

Fig. 2. Amplitude and phase of the reflection coefficient of a normally incident x-polarized plane wave on a Hilbert-curve metamaterial surface above a ground plane at the distance $d=0.536 \mathrm{~mm}$. The size of each Hilbert cell is $1.2 \mathrm{~mm} \times 1.2 \mathrm{~mm}$.

\section{B. Microstrip Dipoles above Hilbert-Curve Metamaterial Surface}

The results for two cases are presented here: i) an electrically short dipole, and ii) a half-wave dipole.

\section{Short Dipole}

Figure 3 shows an electrically small microstrip dipole above a high-impedance surface made of Hilbert inclusions of step order 3. The dipole is assumed to be oriented in y-direction and have a length of $\lambda / 20$ at a frequency of $28 \mathrm{GHz}$. The Hilbert surface in this case consists of a finite array of $11 \times 11$ of Hilbert-curve elements. A single Hilbert element has a footprint of $1.2 \mathrm{~mm} \times 1.2 \mathrm{~mm}$ and its total length, when stretched, is about 10.8 millimeters $(\sim 1 \lambda$ at $28 \mathrm{GHz})$. The distance between adjacent units is set to $0.1174 \mathrm{~mm}$, which is the same as that of the length of a single section in a Hilbertcurve unit. The surface is placed at a distance of $0.536 \mathrm{~mm}$ above the PEC ground-plane, whereas the microstrip dipole is at a height of $0.536 \mathrm{~mm}$ above the Hitbert surface. The dielectric constants of both substrate layers, between the Hilbert surface and the PEC plane and the dipole and Hilbert surface, are assumed to be $\varepsilon_{\mathrm{r}}=1$.

Plots of the real and imaginary parts of the input impedance of the dipole as a function of frequency, and the power gains at specific frequencies within the range, are shown in Figures $4 a$ and $4 b$, respectively. The input reactance remains virtually unchanged over the frequency range while the input resistance increases significantly at 20.5 and $37.5 \mathrm{GHz}$. We note that the latter is close to the resonance of the Hilbert-curve unit at $40 \mathrm{GHz}$. It is noteworthy that the same dipole above a PEC plane, in the absence of the Hilbert surface has an input resistance (radiation resistance) of less than $0.5 \mathrm{ohm}$. An inspection of the power patterns in Figure $4 \mathrm{~b}$ reveals that at 20.5 $\mathrm{GHz}$, the gain has increased to $14.25 \mathrm{dBi}$, which is about $5.5 \mathrm{dBi}$ higher than that of the gain of a dipole in the absence of the Hilbert surface. This increase in gain might be due to the arraying effect of the Hilbert-curve elements and should be studied further in the future.

\section{Half-Wave Dipole}

In the second case, we consider a center-fed $\mathrm{x}$-directed microstrip dipole placed at a distance of 0.375 . The heights of the dipole and the Hilbert surface above the ground-plane are $0.75 \mathrm{~mm}$ and $0.375 \mathrm{~mm}$, respectively. Length of the dipole is $4.09 \mathrm{~mm}$, and is chosen such that the overall system resonates at about $35 \mathrm{GHz}$. All other parameters of the Hilbert surface are set as in the previous case. Figures $5 \mathrm{a}$ and $5 \mathrm{~b}$ show the input impedance and power gains of the antenna, respectively. As can be seen from the zero crossing of the input reactance, the antenna system resonates at about $34.5 \mathrm{GHz}$ resulting in an enhanced input resistance of $75 \mathrm{ohms}$ as compared to less than $20 \mathrm{ohms}$ for an identical dipole in the absence of the Hilbert surface. The corresponding gain, however, is very low in this case. As these figures show, however, both a high input resistance $(\sim 60 \mathrm{ohms})$ and a high matched gain $(\sim 13 \mathrm{dBi})$ can be achieved at an operating frequency of 35 $\mathrm{GHz}$. 


\section{CONCLUSION}

We have used method of moments (MOM) to numerically analyze the radiation characteristics of centerfed microstrip dipoles above a high-impedance metamaterial surface composed of Hilbert-curve inclusions. Numerical results for input impedance, pattern and gain of electrically small as well as half-wave dipoles were presented. In particular, it was shown that the radiation resistance of a microstrip dipole increased significantly at certain frequencies near the resonant frequency of the Hilbert surface. In addition, a significant enhancement of antenna gain was observed at specific frequencies for all dipole sizes analyzed. Results for other dipole sizes and polarization, as well as a parametric study of the input-impedance bandwidth will be given in the presentation.

Acknowledgement

This work was supported in parts by DARPA under Grant No. MDA972-02-1-0022. The content of the information does not necessarily reflect the position or the policy of the Government, and no official endorsement should be inferred.

\section{REFERENCES}

[1] D. Sievenpiper, L. Zhang, R. F. Jimenez Broas, N. G. Alexopolous, and E. Yablonovitch, IEEE Transactions on Microwave Theory and Techniques, Vol. 47, No. 11, pp. 2059-2074, November 1999.

[2] F. Yang and Y. Rahmat-Samii, "Reflection Phase Characterization of an Electromagnetic Band-Gap (EBG) Surface," Proceedings of the 2002 IEEE Antennas and Propagation Society International Symposium, San Antonio, Texas, June 16-21, 2002, pp. 744-747, Vol. 3.

[3] Y. Zhang, J. von Hagen and W. Wiesbeck, "Patch Array as Artificial Magnetic conductors for Antenna Gain Improvement," Microwave and Optical Technology Letters, Vol. 35, No. 3, pp. 172-175, November 5, 2002.

[4] J. McVay, N. Engheta and A. Hoorfar, "High-Impedance Metamaterial Surface using Hilbert-Curve Inclusions," 2002 USNC/URSI National Radio Science Meeting, San Antonio, Texas, June 2002.

[5] Hans Sagan, Space-Filling Curve, Springer-Verlag, NY, 1994.

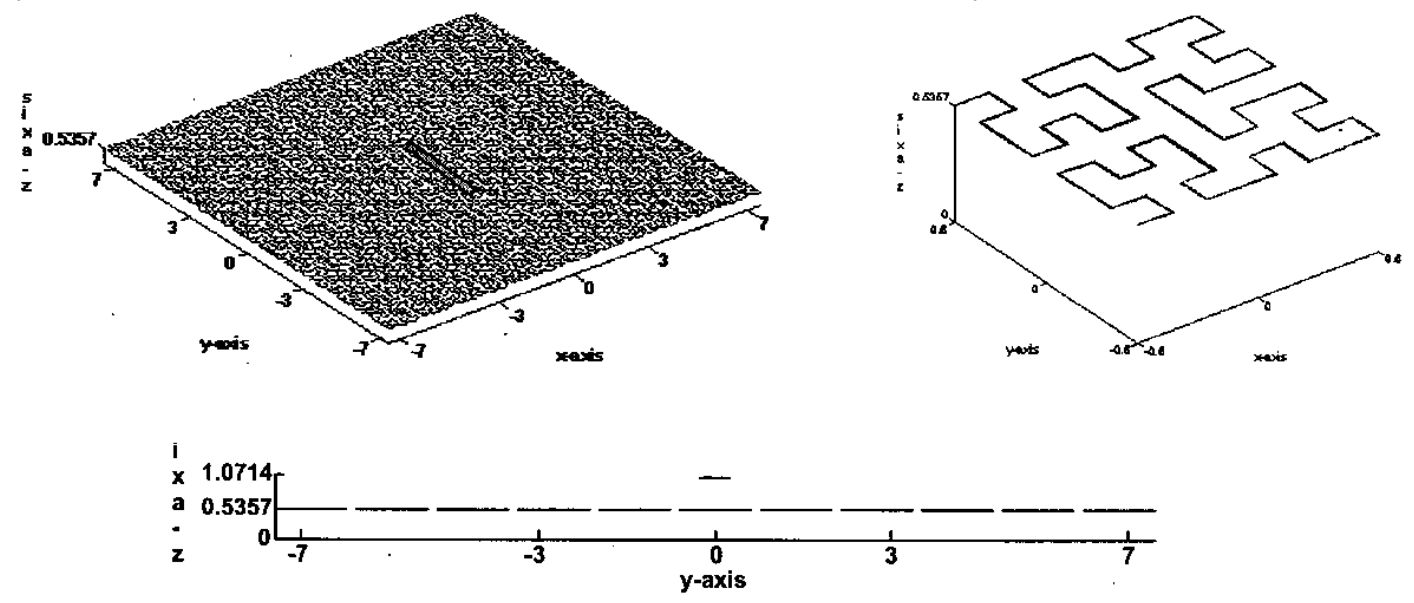

Fig. 3. A microstrip dipole above a high-impedance surface made of Hilbert-curve inclusions of order 3 above a conducting ground plane. 


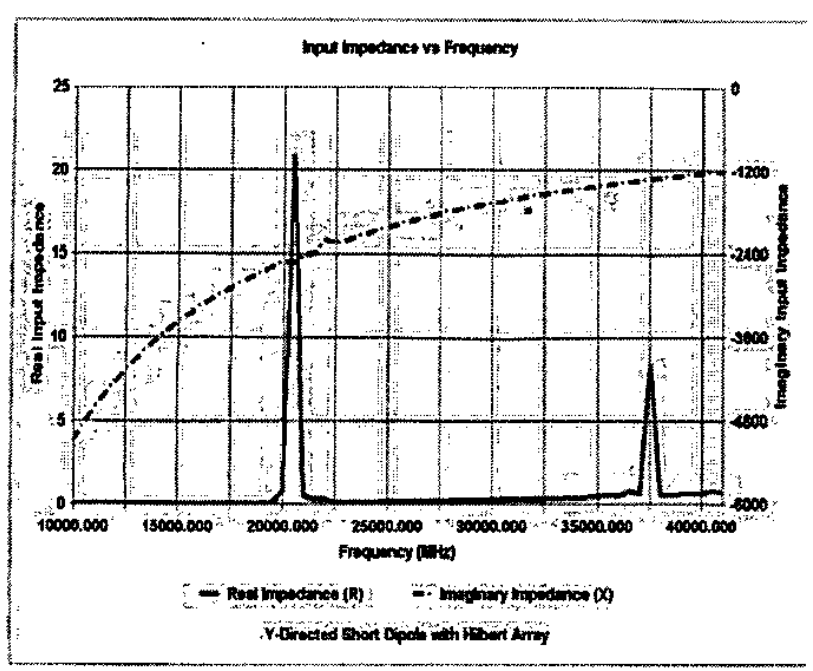

(a)

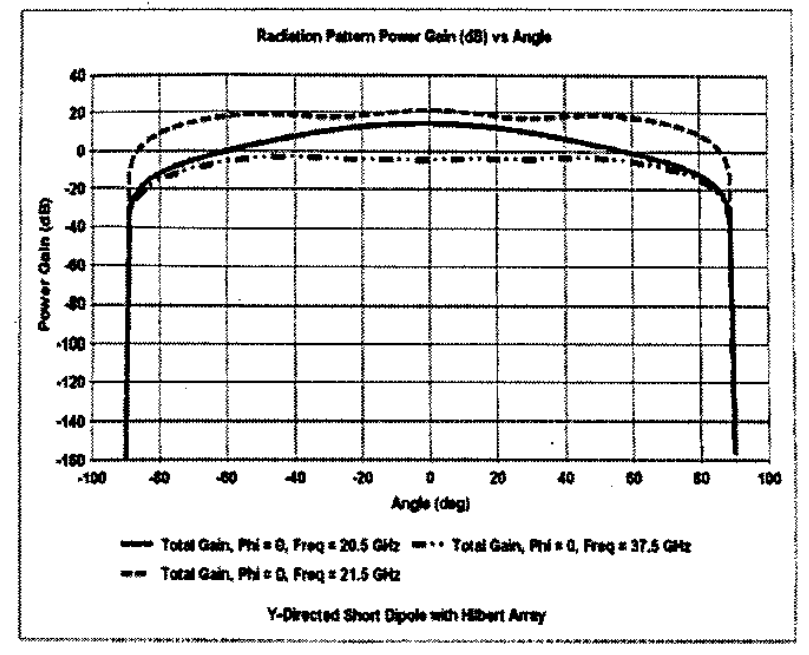

(b)

Fig. 4. Input impedance (a) and gain pattern (b) of a short y-directed dipole above a high-impedance surface made of Hilbert-curve inclusions of order 3 above a conducting ground plane.

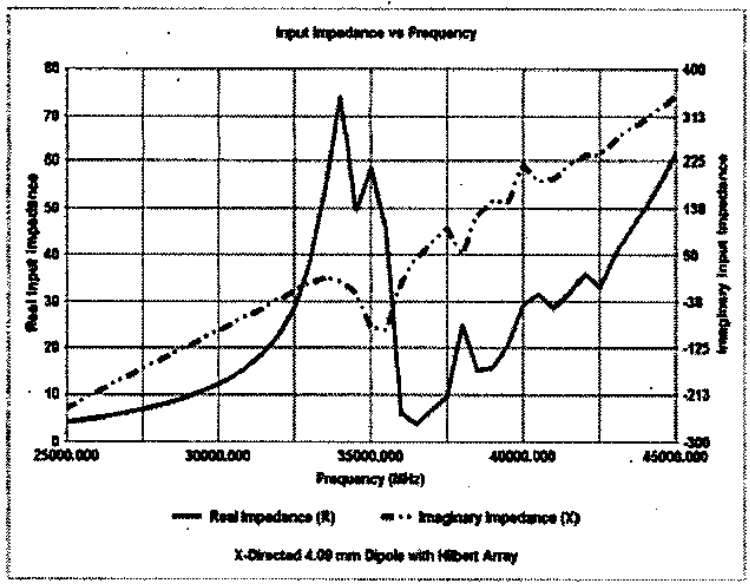

(a)

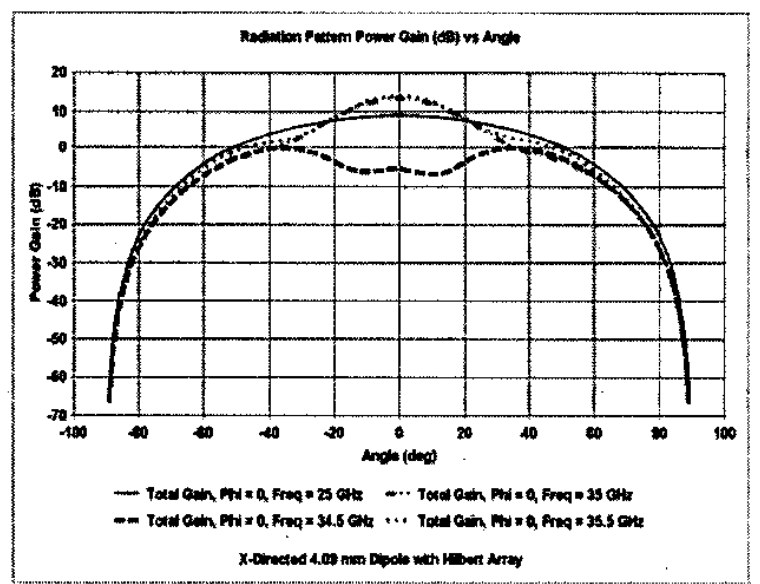

(b)

Fig. 5. Input impedance (a) and gain pattern (b) of a half-wave $x$-directed dipole above a high-impedance surface made of Hilbert-curve inclusions of order 3 above a conducting ground plane. 